\title{
In their own words: patient navigator roles in culturally sensitive cancer care
}

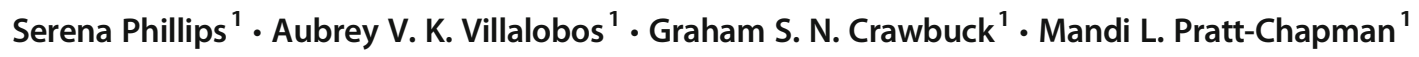

Received: 19 March 2018 / Accepted: 3 August 2018 / Published online: 14 August 2018

(C) The Author(s) 2018

\begin{abstract}
Purpose Patient navigation has emerged as a promising strategy in reducing disparities among diverse cancer patients. However, little is known about navigators' own perspectives on their roles in providing culturally competent care. The purpose of the present study is to describe these self-identified roles.

Methods Data were collected from an online survey with a convenience sample of cancer patient navigators. Using NVivo 10, qualitative content analysis was conducted on free text responses to the question: "In your opinion, what is the role of a patient navigator or nurse navigator in the provision of culturally sensitive care to patients?" Frequencies of each navigator-identified role mentioned were tabulated.

Results Of 294 respondents, $50.7 \%$ ( $n=149)$ provided a response to the question of interest. Respondents described the following 11 interrelated navigator roles in the provision of culturally competent care: (1) assess and understand patient needs, (2) tailor care to patient, (3) build rapport/open communication, (4) facilitate communication between patient and health care team, (5) educate/provide resources to the patient, (6) advocate, (7) self-motivated learning, (8) address barriers to care, (9) involve/meet the needs of family or support people, (10) educate/support health care team, and (11) support patient empowerment in care.

Conclusions Patient navigators are uniquely well-positioned to improve cultural competence of cancer care given their role as liaison to patients and providers. Cancer care settings should use navigators with direct knowledge of patient culture whenever possible; however, communication and cultural competence training is highly recommended for all navigators given the diversity of patient needs.
\end{abstract}

Keywords Patient navigation $\cdot$ Cultural competence $\cdot$ Patient-centered care $\cdot$ Job roles $\cdot$ Cancer

\section{Background}

Culturally, linguistically responsive care is critical to reduce health care disparities [1]. The importance of cultural competence in cancer care has gained deserved attention, with mounting evidence that failures in this area negatively impact care $[2,3]$.

Navigators occupy a strategic role in improving cultural competence in cancer care. Navigators are relative newcomers

The following manuscript reflects original work and has not been submitted for publication at another journal.

Mandi L. Pratt-Chapman

mandi@gwu.edu

1 Institute for Patient-Centered Initiatives and Health Equity, The George Washington University Cancer Center, 2600 Virginia Avenue NW, Suite 300, Washington, DC 20037, USA to the multidisciplinary cancer care team. Navigators come from a variety of backgrounds. Willis et al.'s framework describes similarities and differences across navigator types [4]. Subsequent work has defined major tasks of navigators and articulated core competencies [5-7]. Regardless of background, navigators work to decrease fragmentation and address barriers to care [4-9]. Navigators who are licensed social workers can provide psychosocial support and psychotherapy $[4,5]$. Nurse navigators can coordinate clinical care $[4,5]$. Natale-Pereira and colleagues suggest that navigators can mitigate distrust among racial/ethnic minority patients by establishing rapport with patients, particularly when navigators are from the patient's community [10]. As a "bridge" between the patient's world and the health care system, navigators facilitate communication and identify resources to address language barriers, disparate perspectives on illness and care, and past negative experiences with health care services [10]. For the purposes of navigation evaluation and research, 
experts consider navigator cultural competency to be a core patient-reported outcome measure [11, 12].

Numerous culturally tailored navigation interventions have been developed for diverse groups across the cancer continuum, with promising results [13-21]. These typically involve use of racially or linguistically concordant navigators and/or culturally relevant approaches [13-16]. The Massachusettsbased Cervical Health navigator program's Spanish-speaking Latina immigrant navigator provided in-language education, mitigated barriers to care, and assisted with appointment scheduling. Navigated women had fewer missed colposcopy appointments and lower grade of cervical abnormality [13]. Use of racially concordant professional and peer navigators to provide education and support was also effective in a Harlembased intervention to increase uptake of colonoscopy among African Americans. The program tailored education for participants by using colloquial language, highlighting colorectal cancer disparities for African Americans, and addressing salient barriers [14]. There are also strong examples of culturally tailored navigator programs serving American Indian populations, including the Walking Forward and Native Sisters programs. Effective strategies included involvement of tribal leadership in program development, employment of indigenous navigators, and use of culturally relevant educational materials. Results from these programs included greater mammography adherence and fewer days of treatment interruption among patients receiving curative radiation therapy [22-25].

Despite the importance of cultural competence, navigator programs are often not tailored to address specific cultural needs. Navigators must serve a wide diversity of patients. Furthermore, training for navigators is not uniform and rarely includes strategies to support diverse patients from discordant life experiences [26, 27]. Research on navigators' cultural responsiveness is lacking, particularly from the perspective of navigators themselves. The aim of this study is to report diverse navigators' self-described roles in supporting culturally sensitive cancer care. Results from this study informed a novel educational intervention for cancer care professionals and can inform future educational offerings to better equip navigators to meet the needs of diverse patients [27].

\section{Methods}

\section{Study procedures}

An online survey of cancer navigators was conducted in February 2017 as part of formative work in developing a cultural competence training for multidisciplinary oncology health care professionals. The responses to the survey informed curriculum development of the Together-EquitableAccessible-Meaningful (TEAM) Training [27]. All research procedures were approved by the Institutional Review Board at the George Washington University's (GW) Office of Human Research (\#101646). Informed consent was obtained from all study participants. Participation was solicited using the GW Cancer Center's listservs and social media communication channels. Entry into a random drawing for a \$100 gift card was offered as an incentive. Respondents were eligible to participate if they were over 18 years of age and (1) selfidentified as a navigator of any type, (2) worked with cancer patients or cancer-related services, and (3) worked in the USA. Participants self-administered the 76-item survey via the Research Electronic Data Capture (REDCap) platform. The survey included demographics, work setting, and Likertstyle items measuring attitudes, beliefs, knowledge, and skill in working with diverse patient populations. Three questions were open-ended comment fields, including the question of focus for the present analysis: "In your opinion, what is the role of a patient navigator or nurse navigator in the provision of culturally sensitive cancer care to patients?"

\section{Analytic approach}

Content analysis was conducted on survey comment fields, and codes were enumerated. Qualitative analysis, conducted in NVivo 10, used a pragmatic inductive approach. Coding was completed by research staff members with experience and training in qualitative analytic methods. Authors SP and GC independently coded a subset of responses before comparing preliminary codebooks and then developed a common codebook to apply to the entire set of responses. Passages were coded to multiple codes where appropriate and were not coded if they were nonresponsive to the question of navigator role in provision of culturally sensitive care. SP and GC met regularly to resolve discrepancies, resulting in over $95 \%$ inter-coder agreement. SP, AV, and MPC reviewed the final codebook and codes for conceptual soundness. Quantitative analysis was conducted using Stata 14, including tabulation of participant characteristics and code counts. Logistic regression was used to check sociodemographic differences between respondents versus non-respondents.

\section{Results}

Of the 313 respondents who started the survey, 294 met eligibility criteria. The 149 individuals $(50.7 \%)$ who responded to the question of interest were predominantly female $(97.3 \%)$, white $(77.9 \%)$, heterosexual $(91.3 \%)$, and English-speaking only $(84.6 \%)$. Participants were most frequently 55-64 years old $(32.9 \%)$; worked in a hospital or department within a hospital $(22.8 \%)$, community cancer center $(21.5 \%)$, or hospital-affiliated clinic or outpatient clinic $(20.1 \%)$; worked in a southern state $(35.6 \%)$; and had "quite a bit" $(34.2 \%)$ or "some" (32.9\%) diversity training in the past. Table 1 contains details on participant characteristics. Those who responded to 
Table 1 Oncology patient navigator respondent characteristics $(n=$ 149)

\begin{tabular}{|c|c|}
\hline Characteristic & $\begin{array}{l}\text { Frequency } \\
\text { (percentage) }\end{array}$ \\
\hline \multicolumn{2}{|l|}{ Age } \\
\hline $18-34$ & $19(12.8)$ \\
\hline $35-44$ & $22(14.8)$ \\
\hline $45-54$ & $34(22.8)$ \\
\hline $55-64$ & 49 (32.9) \\
\hline $65+$ & $15(10.1)$ \\
\hline Missing & $10(6.7)$ \\
\hline \multicolumn{2}{|l|}{ Gender identity } \\
\hline Female & $145(97.3)$ \\
\hline Male & $4(2.7)$ \\
\hline \multicolumn{2}{|l|}{ Race/ethnicity } \\
\hline White & $116(77.9)$ \\
\hline Black or African American & $11(7.4)$ \\
\hline Hispanic, Latino, or Spanish origin & $5(3.4)$ \\
\hline Asian & $2(1.3)$ \\
\hline Native Hawaiian or other Pacific Islander & $2(1.3)$ \\
\hline Middle Eastern or North African & $2(1.3)$ \\
\hline American Indian or Alaska Native & $1(0.7)$ \\
\hline Multiple groups & $8(5.4)$ \\
\hline Missing & $2(1.3)$ \\
\hline \multicolumn{2}{|l|}{ Language ability } \\
\hline English only & $126(84.6)$ \\
\hline Multilingual & $21(14.1)$ \\
\hline Missing & $2(1.3)$ \\
\hline \multicolumn{2}{|l|}{ Sexual orientation } \\
\hline Straight & $136(91.3)$ \\
\hline Gay or lesbian & $5(3.4)$ \\
\hline Bisexual & $5(3.4)$ \\
\hline Missing & $3(2.0)$ \\
\hline \multicolumn{2}{|l|}{ Practice setting } \\
\hline Hospital or department within hospital & $34(22.8)$ \\
\hline Community cancer center & $32(21.5)$ \\
\hline Hospital-affiliated clinic or outpatient clinic & $30(20.1)$ \\
\hline Academic cancer center & $23(15.4)$ \\
\hline Nonprofit organization & $13(8.7)$ \\
\hline Community health center & $3(2.0)$ \\
\hline Other & $14(9.4)$ \\
\hline \multicolumn{2}{|l|}{ Geographic region } \\
\hline South & $53(35.6)$ \\
\hline North & $31(20.8)$ \\
\hline West & $28(18.8)$ \\
\hline Midwest & $25(16.8)$ \\
\hline Missing & $12(8.1)$ \\
\hline \multicolumn{2}{|l|}{ Past diversity training } \\
\hline A little & $21(14.1)$ \\
\hline Some & $49(32.9)$ \\
\hline Quite a bit & $51(34.2)$ \\
\hline A lot & $28(18.8)$ \\
\hline
\end{tabular}

the question of interest tended to be older $(p=0.01)$ than those who did not, but did not statistically significantly differ on race, previous diversity training amount, geographic region, or foreign language ability.

\section{Patient navigator roles in cultural competence}

Text examples included below are not edited for grammar. A small number of responses $(n=12)$ were too vague to be meaningfully coded. Navigators identified 11 interrelated ways through which navigators perceived their facilitation of culturally competent care. Code counts were too small to meaningfully distinguish demographic heterogeneity across themes.

\section{Assess and understand patient needs}

Navigators frequently described assessing and understanding patients' unique needs as important $(n=51)$. Navigators described cultural needs as one of many important aspects to consider in providing appropriate, patient-centered care:

Just as important as every other service we provide. Culture, gender, race, religion, sexual orientation, disease staging, support systems in place, family dynamics - all of these make up the total person and help us to understand their strengths and challenges. The more we know, the better we can treat the patient. (female, age 65, New York)

Navigators perceived culture as an important consideration when assessing individual patient beliefs and needs:

It is incumbent upon Navigators to assess each patients cultural preferences, identity and awareness level and provide care...that is sensitive to...each patient individually. (male, age 32, Tennessee)

The role is to initially identify the need for culturally sensitive services by the patient and document those needs and recommend how to meet those needs, i.e., translation services, consideration of religious needs, recognition of sexual orientation and related risks, including appropriate support people, etc. (female, age 62, Montana)

These assessments equipped navigators with the information necessary to support tailored services for each patient.

\section{Tailor care to patient}

Navigators described their important role of tailoring care and services to each patient $(n=44)$ :

Learn to which culture they most identify, try and find support groups, materials, etc. of that appropriate culture. (male, age 38, Michigan)

[M] eet the person where they are as an individual who happens to be of a unique culture and help them navigate the health care system according to their goals/ needs (female, [age blank], Maryland)

To be hyper vigilant for opportunities in all areas of patient care to provide culturally sensitive care. 
Sometimes, it is as simple as food, other times it's privacy. (female, age 76, Georgia)

Navigators described patients as individuals who identified with particular groups and sought to adjust services to be most appropriate for each patient.

\section{Build rapport/open communication}

Navigators reported an important role in building rapport with patients $(n=38)$, especially as the first point of contact in some settings. They noted the importance of their own behavior when interacting with patients in order to create a good first impression on behalf of the health care team:

Since we are often one of the first points of contact, we have a responsibility to provide patients with a culturally sensitive experience that fosters a trusting relationship with our practitioners and support staff. It will either go in a good direction from there, or can be disastrous if we are insensitive. (female, age 56, New Jersey)

Navigators also described the importance of creating spaces where patients were able to disclose information important to their care without fear of judgment:

[T]o speak to them as they are and accept them as they are so they are not afraid to be who they are and share fully (female, age 71, Georgia)

I believe the role of the Navigator is very important because the patient typically feels more comfortable to talk freely with myself as the Navigator. A lot of patients have white coat syndrome and don't want to speak to their physician as candidly. (female, age 48, Florida)

To build trust for individualized assessment, navigators recognized active listening and approachability as important skills.

\section{Facilitate communication between patient and health care team}

Navigators described serving as intermediaries between patients and providers $(n=35)$. They transmitted information in both directions and tried to promote common understanding:

The role of a navigator is to be a conduit between the patient/family and the treatment team. I do think the role lends itself well to be a 'culture translator.' (female, age 55, Washington)

A navigator can help mediate between culturally 'different' and standard Western medicine to reach a treatment plan the patient can accept (female, [age blank], California)
Figuring out and communicating what is and isn't understood between patient and medical team. (female, age 33, Ohio)

In particular, navigators brought patient cultural needs and beliefs to the attention of the rest of the health care team:

Helping the doctor to be aware of the cultural issues. Typically as the navigator, I spend more time with the patients, and know more of their personal issues and things going on outside of their cancer diagnosis and bring them to the attention of the [doctor]. (female, age 40, Texas) To bring in the whole picture in a manner that the health care team respects cultural choices in care (female, [age blank], North Carolina)

Navigators reported skills to communicate effectively with both patients and providers. They helped resolve misunderstandings, bridge the patient and biomedical culture, ensure interpretation services, and facilitate care solutions acceptable to patients. One navigator described serving as a single point of contact to inform providers of patients' cultural preferences as care transitions occurred.

\section{Educate/provide resources to patient}

Navigators described their role in educating patients $(n=29)$, and the importance of doing so in a culturally and linguistically appropriate way:

We are the first line of communication and education for
most patients, thus it is essential that we provide care
that is culturally sensitive. (female, age 51, Alabama)
Our role is to educate patients on the recommendations
made by the physicians while taking into account the
patients beliefs. (female, age 42, New York)

After discovering the patient's cultural perspectives and linguistic needs, navigators reported tailoring resources to be most relevant and responsive to patient needs.

\section{Advocate}

Some navigators described "advocating" for patients $(n=20)$. Though related to the "conduit" role, advocacy implies a more active role in speaking out to support patient perspectives, preferences, or needs:

To help understand personal and cultural preferences, and to advocate for the patient's preferences with the care team. (female, age 26, Utah)

We need to ensure that ALL health care needs are met without discrimination. We may act as a liaison between 
the patient and the health care team to ensure that their beliefs are communicated to the health care team and that we work together to meet the patient's needs. (female, age 47, [state blank])

Some navigators described their advocacy role as not being unique to the issue of culture:

The overall role is the same, be an advocate for your client and ensure they receive appropriate and timely care. (female, age 42, Maryland)

\section{Self-motivated learning}

Navigators described the importance of self-motivated learning to improve their capacity to support patients and other staff to ensure high-quality care $(n=19)$ :

Utilizing the internet, taking courses online and becoming culturally sensitive to others has helped bridge the gap in these instances and have improved communication which have led to higher patient satisfaction and care. (female, age 42, Georgia)

Learning as much as I can about the culture and sharing it with the nurses who are actively providing care. (female, age 54, Kentucky)

\section{Address barriers to care}

Addressing barriers (including cultural barriers) to care was reported as a core part of the navigator job $(n=$ 18):

I feel we can greatly assist in the provision of culturally sensitive cancer care to patients. It's another barrier to overcome and that's what our job entails - breaking down barriers to ensure patients get care. (female, age 35, District of Columbia)

Navigators also mentioned the importance of acting in a culturally sensitive manner themselves to avoid creating new barriers:

Patient or nurse navigators need to be culturally sensitive in working with individuals. If you are unable to provide culturally sensitive care to patients it no longer becomes patient centered care and that can cause future barriers for patients. (female, age 29, Pennsylvania)

\section{Involve/meet the needs of family or support people}

Twelve navigators mentioned family. Some respondents simply included the family alongside the patient in discussing perspectives, needs, and care:

The role of the patient navigator is to be extremely sensitive to the needs of the patient and family members. (female, age 49, Colorado)

Others discussed the navigator's role in understanding the patient's family and support system, and including people important to the patient in the care process:

We should include family members and other supports if the patient has expressed that they are an important part of their life, and decision making. (female, age 36, Pennsylvania)

\section{Educate/support health care team}

Navigators described having a role in educating colleagues about general diversity and culture, not specific to individual patient cases $(n=11)$ :

[T]o educate other team members on cultural preferences and how they relate to patient care (female, age 44, Kentucky)

To increase sensitivity by staff to different beliefs and cultures, help staff identify their own hotspots and hopefully deal with these issues, teach staff about different cultures/beliefs and model this behavior. Finally, learn as much as I can so I can help others. (female, age 56, Maryland)

Navigators described serving as a resource among staff in modeling culturally competent behavior, teaching others about specific cultures and the significance of culture, and encouraging others to provide culturally sensitive care.

\section{Support patient empowerment in care}

Navigators described supporting patient empowerment $(n=7)$ in their care. Distinct from the advocacy role, supporting patient empowerment created space for the patient to selfadvocate:

To learn cultural medical beliefs in general and to hear from each patient his/her own personal beliefs. By doing this, the patient or nurse navigators can help to empower patients. (female, age 68, Wisconsin) 
To provide as much understanding as possible so that patient may take an active role in decision making and compliance. (female, age 63, Tennessee)

Navigators helped empower patients to share their beliefs, make decisions, and communicate preferences with others.

\section{Discussion}

It is striking yet unsurprising that navigators describe their roles in a way that aligns with the core functions of navigators as described in the literature [5, 6, 28]. The founder of the field of navigation, Dr. Harold Freeman, describes navigation as "a patient-centered health care service delivery model" [9]. Epner and Baile eschew outdated "categorical" approaches to cultural competence that stereotype patients based on cultural group membership, and suggest that patient-centered strategies of negotiating cross-cultural communication are key to culturally competent care [29]. Similarly, in a discussion of patient centeredness, cultural competence, and health care quality, Saha and colleagues conclude that "many of the core features of cultural competence and patient centeredness are the same" [30]. Although each has unique elements and different foci, essential elements of both patient centeredness and cultural competence at the interpersonal level include the ability to view each patient holistically as unique individuals, build rapport, explore patient worldviews and perspectives of illness, practice non-judgment, tailor care to patient needs, and negotiate agreement about treatment. Thus, patient centeredness may provide a strategy to address inequities by promoting patient rapport and affective support [30].

The interrelation between cultural competence and patientcentered care may explain why several respondents described their role in supporting cultural competence as an integrated aspect of their inherently patient-centered navigation responsibilities. Many typical activities such as assessment, rapportbuilding, addressing barriers to care, and facilitating communication are patient centered in nature and are not exclusive to issues of culture $[6,31,32]$. For instance, navigators may take the time to gain a deep understanding of patient perspectives of their illness. These patient-centered actions begin dialogues about culture-informed viewpoints critical to the provision of culturally competent care.

Findings from this study should inform future educational offerings for navigators of all types. Since navigators play critical roles in assessing needs and troubleshooting barriers to care, tailoring care, bridging communication between patients and the health care team, supporting patient empowerment, advocating for patients, and providing resources and support to patients and caregivers, strategies to perform these functions should be included in training and professional development offerings. Current navigation trainings, tools, and resources have been recently summarized [26]. One training that is available online and at no cost is the GW Cancer Center Oncology Patient Navigator Training: The Fundamentals [33]. Additionally, the GW Cancer Center TEAM Training was piloted in 2017 to improve cultural responsiveness among multidisciplinary teams and support system-level health equity improvements, leveraging data from the present study to inform the curriculum [27]. Finally, a new resource was created to help patients bring forward their individual care priorities through the TEAM Training project: "I Want You to Know" patient cards, which are available in English, Spanish, and simplified Chinese at http://bit.ly/ TEAMPatientCards.

\section{Limitations}

The study used a web-based convenience sample of volunteer participants and only half of survey respondents answered the open-response question, introducing selection bias. Participants and findings may not be generalizable to the larger population of U.S. oncology navigators.

Content analysis of survey comment fields allows for enumerating frequency of ideas among a large sample (although this number cannot be considered an estimate of prevalence) while simultaneously eliciting unanticipated perspectives and preserving the respondent's voice [34]. However, a limitation of the study typical of this methodology is that responses in the open-response field are brief and lack the depth and detail characteristic of other qualitative data. Furthermore, some of the open-response code counts have low frequencies, making more complex quantitative analyses difficult. Future qualitative research can build upon findings through in-depth interviews with navigators to develop a deeper and more nuanced understanding of perceived roles in cultural competency. Future quantitative studies could use the navigator roles identified in the present study to survey a larger sample of navigators to explore patterns in self-reported roles by respondent demographic or practice characteristics.

\section{Practice implications}

This study suggests that training in patient communication and culturally competent practice is important for navigators, regardless of background, given their unique role as liaison and patient advocate on the multidisciplinary team. As several respondents noted, navigators often function as spokespeople for the health care team as the first and primary point of contact for patients. This study also highlights the critical role of navigators in provision of culturally responsive, patientcentered cancer care.

Funding information This research was supported by the Pfizer Foundation. Contents are solely the responsibility of the authors and do 
not necessarily represent the official views of the Pfizer Foundation. REDCap infrastructure that made this project possible was partially supported by Award Number UL1TR001876 from the NIH National Center for Advancing Translational Sciences. Its contents are solely the responsibility of the authors and do not necessarily represent the official views of the National Center for Advancing Translational Sciences or the National Institutes of Health.

\section{Compliance with ethical standards}

Ethical approval All procedures performed in studies involving human participants were in accordance with the ethical standards of the institutional and/or national research committee and with the 1964 Helsinki declaration and its later amendments or comparable ethical standards.

Conflict of interest The authors declare that they have no conflict of interest.

Open Access This article is distributed under the terms of the Creative Commons Attribution-NonCommercial 4.0 International License (http:// creativecommons.org/licenses/by-nc/4.0/), which permits any noncommercial use, distribution, and reproduction in any medium, provided you give appropriate credit to the original author(s) and the source, provide a link to the Creative Commons license, and indicate if changes were made.

\section{References}

1. Fisher TL, Burnet DL, Huang ES, Chin MH, Cagney KA (2007) Cultural leverage: interventions using culture to narrow racial disparities in health care. Med Care Res Rev 64:243S-282S. https:// doi.org/10.1177/1077558707305414

2. Tucker CM, Marsiske M, Rice KG, Nielson JJ, Herman K (2011) Patient-centered culturally sensitive health care: model testing and refinement. Health Psychol 30:342-350. https://doi.org/10.1037/ a0022967

3. Kagawa-Singer M, Valdez Dadia A, Yu MC, Surbone A (2010) Cancer, culture, and health disparities: time to chart a new course? CA Cancer J Clin 60:12-12-39. doi: https://doi.org/10.3322/caac. 20051

4. Willis A, Reed E, Pratt-Chapman M, Kapp H, Hatcher E, Vaitones V, Collins S, Bires J, Washington E (2013) Development of a framework for patient navigation: delineating roles across navigator types. J Cancer Surviv 4:20-26

5. Wells KJ, Valverde P, Ustjanauskas AE, Calhoun EA, Risendal BC (2018) What are patient navigators doing, for whom, and where? A national survey evaluating the types of services provided by patient navigators. Patient Educ Couns 101:285-294. https://doi.org/10. 1016/j.pec.2017.08.017

6. Pratt-Chapman M, Willis A, Masselink L (2015) Core competencies for oncology patient navigators. J Oncol Navig Surviv 6:16-21

7. Oncology Nursing Society (2015) Oncology nursing core competencies. https://www.ons.org/practice-resources/competencies. Accessed 16 March 2018

8. Freund K, Paskett ED, Young GS, Battaglia TA, Calhoun E, Darnell JS, Dudley DJ, Fiscella K, Hare ML, Murray DM, LaVerda N, Levine P, Lee J, Roetzheim RG, Patierno SR, Raich PC, Whitley EM, Simon M, Snyder FR, Warren-Mears V, Winters $P$ (2014) Impact of patient navigation on timely cancer care: the Patient Navigation Research Program. J Natl Cancer Inst 106. https://doi.org/10.1093/jnci/dju115
9. Freeman HP (2012) The origin, evolution, and principles of patient navigation. Cancer Epidemiol Biomark Prev 21:1614-1617

10. Natale-Pereira A, Enard KR, Nevarez L, Jones LA (2011) The role of patient navigators in eliminating health disparities. Cancer 117: 3543-3552. https://doi.org/10.1002/cncr.26264

11. Fiscella K, Ransom S, Jean-Pierre P, Cella D, Stein K, Bauer JE, Crane-Okada R, Gentry S, Canosa R, Smith T, Sellers J, Walsh K (2011) Patient-reported outcome measures suitable to assessment of patient navigation. Cancer 117:3603-3617

12. Pratt-Chapman M, Patierno S, Simon MA, Patterson AK, Risendal BC (2011) Survivorship navigation outcome measures: a report from the ACS Patient Navigation Working Group on Survivorship Navigation. Cancer 117:3575-3584

13. Percac-Lima S, Benner CS, Lui R, Aldrich LS, Oo SA, Regan N, Chabner BA (2013) The impact of a culturally tailored patient navigator program on cervical cancer prevention in Latina women. $\mathrm{J}$ Women's Health 22:426-431. https://doi.org/10.1089/jwh.2012. 3900

14. Jandorf L, Cooperman JL, Stossel LM, Itzkowitz S, Thompson HS, Villagra C, Thélémaque LD, McGinn T, Winkel G, Valdimarsdottir H, Shelton RC, Redd W (2013) Implementation of culturally targeted patient navigation system for screening colonoscopy in a direct referral system. Health Educ Res 28:803-815. https://doi.org/ 10.1093/her/cyt003

15. Fischer SM, Cervantes L, Fink RM, Kutner JS (2015) Apoyo con Cariño: a pilot randomized controlled trial of a patient navigator intervention to improve palliative care outcomes for Latinos with serious illness. J Pain Symptom Manag 49:657-665. https://doi.org/ 10.1016/j.jpainsymman.2014.08.011

16. Fischer SM, Sauaia A, Kutner JS (2007) Patient navigation: a culturally competent strategy to address disparities in palliative care. J Palliative Med 10:1023-1028. https://doi.org/10.1089/jpm.2007. 0070

17. Percac-Lima S, Ashburner JM, Bond B, Oo SA, Atlas SJ (2013) Decreasing disparities in breast cancer screening in refugee women using culturally tailored patient navigation. J Gen Intern Med 28: 1463-1468. https://doi.org/10.1007/s11606-013-2491-4

18. Percac-Lima S, Grant RW, Green AR, Ashburner JM, Gamba G, Oo S, Richter JM, Atlas SJ (2009) A culturally tailored navigator program for colorectal cancer screening in a community health center: a randomized, controlled trial. J Gen Intern Med 24:211217. https://doi.org/10.1007/s11606-008-0864-x

19. Mollica MA, Nemeth LS, Newman SD, Mueller M, Sterba K (2014) Peer navigation in African American breast cancer survivors. Patient Relat Outcome Meas 2014:131-144

20. Maxwell AE (2010) Peer navigation improves diagnostic follow-up after breast cancer screening among Korean American women: results of a randomized trial. Cancer Causes Control 21:1931-1940

21. DeGroff A, Schroy PC III, Morrissey KG, Slotman B, Rohan EA, Bethel J, Murillo J, Ren W, Niwa S, Leadbetter S, Joseph D (2017) Patient navigation for colonoscopy completion: results of an RCT. Am J Prev Med 53:363-372

22. Whop LJ, Valery PC, Beesley VL, Moore SP, Lokuge K, Jacka C, Garvey G (2012) Navigating the cancer journey: a review of patient navigator programs for Indigenous cancer patients. Asia-Pac J Clin Oncol 8:e89-e96. https://doi.org/10.1111/j.1743-7563.2012.01532.x

23. Burhansstipanov L, Bad Wound D, Capelouto N, Goldfarb F, Harjo L, Hatathlie L, Vigil G, White M (1998) Culturally relevant 'navigator' patient support: the Native Sisters. Cancer Pract 6:191-194

24. Molloy K, Reiner M, Ratteree K, Cina K, Helbig P, Miner R, Elk DL, Tail CS, Sparks S, Tiger L, Esmond S, Petereit DG (2007) Patient navigation \& cultural competency in cancer care. Oncology Issues 22:38-41. https://doi.org/10.1080/10463356. 2007.11883359

25. Guadagnolo BA, Boylan A, Sargent M, Koop D, Brunette D, Kanekar S, Shortbull V, Molloy K, Petereit DG (2011) Patient 
navigation for American Indians undergoing cancer treatment: utilization and impact on care delivery in a regional healthcare center. Cancer 117:2754-2761. https://doi.org/10.1002/cncr.25823

26. Pratt-Chapman M, Burhansstipanov L (2018) Navigation training, tools and resources. In: Shockney L (ed) Team-based oncology care: the pivotal role of oncology navigation. Springer International Publishing, pp 315-334

27. The George Washington University Cancer Center, (2017) TEAM: Together-Equitable-Accessible-Meaningful. https://cancercenter. gwu.edu/for-health-professionals/team-together-equitableaccessible-meaningful

28. Rohan EA, DeGroff A, Slotman B, Morrissey KG, Murillo J, Schroy P (2016) Refining the patient navigation role in a colorectal cancer screening program: results from an intervention study 14 : $1371-1378$

29. Epner DE, Baile WF (2012) Patient-centered care: the key to cultural competence. Ann Oncol 23:33-42. https://doi.org/10.1093/ annonc/mds086
30. Saha S, Beach MC, Cooper LA (2008) Patient centeredness, cultural competence and healthcare quality. J Natl Med Assoc 100: 1275-1285. https://doi.org/10.1016/S0027-9684(15)31505-4

31. Gunn CM, Clark JA, Battaglia TA, Freund KM, Parker VA (2014) An assessment of patient navigator activities in breast cancer patient navigation programs using a nine-principle framework. Health Serv Res 49:1555-1577. https://doi.org/10.1111/1475-6773.12184

32. Rasulnia M, Sih-Meynier R (2017) The roles and challenges of oncology navigators: a national survey. J Oncol Navig Surviv 8

33. The George Washington University Cancer Center, (2015) Oncology patient navigator training: the fundamentals. https:/ smhs.gwu.edu/cancergate/best-practice/oncology-patientnavigator-training-fundamentals. Accessed 5 July 2018

34. Stoneman P, Sturgis P, Allum N (2013) Exploring public discourses about emerging technologies through statistical clustering of openended survey questions. Public Underst Sci 22:850-868 